\title{
The Simplex Reminiscent of Sperner's Lemma
}

\author{
A. Ramesh Kumar ${ }^{1}$ and G. Kavitha ${ }^{2}$ \\ Head, Department of Mathematics, Srimad Andavan Arts \& Science College, Trichy ${ }^{1}$ \\ Asst. Professor, Kongunadu College of Engineering and Technology, Tholurpatti, Thottiam ${ }^{2}$
}

\begin{abstract}
We prove three results about colorings of the simplex reminiscent of Sperner's Lemma in hardness of approximation and fair division. First, let $\mathrm{V}_{\mathrm{k}, \mathrm{q}}=\left\{\mathrm{v} \in \square_{+}^{\mathrm{k}}: \sum_{\mathrm{i}=1}^{\mathrm{k}} \mathrm{v}_{\mathrm{i}}=\mathrm{q}\right\}$ and $\mathrm{E}_{\mathrm{k}, \mathrm{q}}=\left\{\left\{\mathrm{a}+\mathrm{e}_{1}, \mathrm{a}+\mathrm{e}_{2}, \ldots, \mathrm{a}+\mathrm{e}_{\mathrm{k}}\right\}: \mathrm{a} \in \square_{+}^{\mathrm{k}}: \sum_{\mathrm{i}=1}^{\mathrm{k}} \mathrm{a}_{\mathrm{i}}=\mathrm{q}-1\right\}$. Then for every Sperner-admissible labeling $\left(1: \mathrm{V}_{\mathrm{k}, \mathrm{q}} \rightarrow[\mathrm{k}]\right.$ such that $\mathrm{v}_{\mathrm{l}(\mathrm{v})}>0$ for each $\left.\mathrm{v} \in \mathrm{V}_{\mathrm{k}, \mathrm{q}}\right)$, there are at least $\left(\begin{array}{c}\mathrm{q}+\mathrm{k}-3 \\ \mathrm{k}-2\end{array}\right)$ non-monochromatic hyperedges in $\mathrm{E}_{\mathrm{k}, \mathrm{q}}$. This implies an optimal Unique-Games hardness of (k-1- $\in$ )approximation for the Hypergraph Labeling with Color Lists problem in a k-uniform hypergraph $\mathrm{H}=(\mathrm{V}, \mathrm{E})$ with color lists $\mathrm{L}(\mathrm{v}) \subseteq[\mathrm{k}] \forall \mathrm{v} \in \mathrm{V}$. To prove labeling $\mathrm{l}(\mathrm{v}) \in \mathrm{L}(\mathrm{v})$ that minimizes the number of non-monochromatic hyperedges. We also show that a (k - 1)-approximation can be achieved. Second, we show that in contrast to Sperner's Lemma, there is a Sperner-admissible labeling of $\mathrm{V}_{\mathrm{k}, \mathrm{q}}$ such that every hyperedge in $\mathrm{E}_{\mathrm{k}, \mathrm{q}}$ contains at most 4 colors. We present an interpretation of this statement in the context of fair division : There is a preference function on $\Delta_{\mathrm{k}, \mathrm{q}}=$ $\left\{\mathrm{x} \in \square_{+}^{\mathrm{k}}: \sum_{\mathrm{i}=1}^{\mathrm{k}} \mathrm{x}_{\mathrm{i}}=\mathrm{q}\right\}$ such that for any division of $\mathrm{q}$ units of a resource, $\left(\mathrm{x}_{1}, \mathrm{x}_{2}, \ldots, \mathrm{x}_{\mathrm{k}}\right) \in \Delta_{\mathrm{k}, \mathrm{q}}$ such that $\sum_{\mathrm{i}=1}^{\mathrm{k}}\left[\mathrm{x}_{\mathrm{i}}\right]=\mathrm{q}-1$, at most 4 players out of $\mathrm{k}$ are satisfied.
\end{abstract}

Keywords: Sperner's Lemma, hyper graph label and hyper graph label and hyper graph colouring. MSC Code : $05 \mathrm{C} 65,05 \mathrm{C} 90$

\section{INTRODUCTION}

We investigate hypergraph labeling problems of the following kind. Hypergraph Labeling with Color Lists: Given a hypergraph $\mathrm{H}=(\mathrm{V}, \mathrm{E})$ with weights $\mathrm{w}(\mathrm{e}), \mathrm{e} \in \mathrm{E}$ and color lists $\mathrm{L}(\mathrm{v}) \subseteq[\mathrm{k}], \mathrm{v} \in \mathrm{V}$, find a labeling $\mathrm{l}(\mathrm{v}) \in$ $\mathrm{L}(\mathrm{v})$ for each $\mathrm{v} \in \mathrm{V}$ that minimizes the total weight of non-monochromatic hyperedges.

This problem (is an equivalent form with assignment costs, referred to as Hypergraph Labeling) was introduced in [1] as a generalization of Uniform Metric Labeling [5], to incorporate relationships between multiple elements. Hypergraph Labeling with Color Lists can be cast in a more general framework involving submodular functions, as follows.

Submodular Labeling with Color Lists: Given a submodular function $\mathrm{f}: 2^{\mathrm{V}} \rightarrow \square$, and color lists $\mathrm{L}(\mathrm{v}) \subseteq$ $[\mathrm{k}], \mathrm{v} \in \mathrm{V}$, find a labeling $\mathrm{l}(\mathrm{v}) \in \mathrm{L}(\mathrm{v})$ that minimizes $\sum_{\mathrm{i}=1}^{\mathrm{k}} f\left(l^{-1}(\mathrm{i})\right)$.

Partitioning problems of this type have been investigated recently in $[2,3,6,5]$. The main result of [2] is a 2approximation for Submodular Multiway Partition, a special case of Submodular Labeling with Color Lists where the color lists are either singletons ("terminals") or equal to $[\mathrm{k}]$ (unrestricted). This captures problems such as
Hypergraph Multiway Cut and Node-weighted Multiway Cut (see [2]), where the color lists are similarly restricted to be singletons or $[\mathrm{k}]$. Without this restriction, Submodular Labeling with Color Lists does not admit factors better than $\log n$, by a simple reduction from Set Cover [1]. An $\mathrm{O}(\mathrm{k} \log \mathrm{n})$-approximation for Submodular Labeling with Color Lists has been given in [3].

For Hypergraph Labeling, [1] gave a $\Delta$-approximation when all hyperedges have size at most $\Delta$. This generalizes a 2-approximation for Uniform Metric Labeling [5] which corresponds to the $\Delta=2$ case. On the hardness side, the strongest negative result was a hardness of $(2-\epsilon)$ approximation assuming the Unique Games Conjecture[6].

In [3], a statement somewhat reminiscent of Sperner's Lemma was conjectured, which would imply an integrality gap and also a hardness of $(\mathrm{k}-1-\in)$-approximation under the UGC[4], for Hyper-graph Labeling with Color Lists on k-uniform hypergraphs with label set [k]. This conjecture gives a lower bound on the number of nonmonochromatic hyperedges for any feasible labeling of a certain hypergraph $\mathrm{H}_{\mathrm{k}, \mathrm{q}}$ embedded in the simplex. This statement was proved for $\mathrm{k}=3$ in [3]; it implies a UniqueGames hardness of (2 - $\in$ )-approximation for Hypergraph Labeling with Color Lists on 3-uniform hypergraphs with label set [2]. 


\section{MODELS}

Assuming the Unique Games Conjecture, there is no $(\mathrm{k}-1$ - $\in$ )-approximation for Hypergraph Labeling with Color Lists on k-uniform hypergraphs with label set $[\mathrm{k}]$. A $\Delta$ approximation algorithm, where $\Delta$ is the maximum size of a hyperedge, was known for this problem [1]; we show that the same algorithm also gives a $(\mathrm{k}-1)$ approximation. Thus, we determine the optimal approximability of Hypergraph Labeling with Color Lists in terms of the label set size.

This result motivates us to consider other coloring questions related to Sperner's Lemma and the conjecture of [3]. We prove that in contrast to Sperner's Lemma, the hypergraph $\mathrm{H}_{\mathrm{k}, \mathrm{q}}$ defined in [3] can be labeled in such a way that each hyperedge uses at most 4 colors. This implies in particular that the hardness result for Hypergraph Labeling with Color Lists holds even for hypergraphs with a feasible labeling such that each hyperedge uses at most 4 colors.

We also give an interpretation of this result in the setting of fair division[8]. Our result shows that for a certain restricted variant of fair division, not only is it impossible to satisfy all players but in fact all players except four will be always unsatisfied.

Further, we consider simplicial subdivisions and ask what fractional labelings are possible for subdivisions of the simplex. We show that for sufficiently fine subdivisions, there is a fractional (Sperner-admissible) labeling that uses local labeling using at most 2 colors for each hyperedge. In contrast, by Sperner's Lemma, for any admissible labeling there is a hyperedge with all $\mathrm{k}$ colors. This has consequences for the following problem.

\subsection{Example}

Hypergraph j-Colors-Avoiding Labeling with Color Lists: Given a hypergraph $\quad \mathrm{H}=(\mathrm{V}, \mathrm{E})$ with color lists $\mathrm{L}(\mathrm{v}) \subseteq[\mathrm{k}] ; \mathrm{v} \in \mathrm{V}$, find a labeling $\mathrm{l}(\mathrm{v}) \in \mathrm{L}(\mathrm{v})$ for each $\mathrm{v} \in \mathrm{V}$ that minimizes the number of hyperedges containing at least $\mathrm{j}$ distinct colors.

In particular, for $\mathrm{j}=\mathrm{k}$ we try to avoid hyperedges containing all $\mathrm{k}$ colors; we call this problem Hypergraph Rainbow-Avoiding Labeling with Color Lists. Our result implies that a natural LP for this problem cannot distinguish between instances that can be labeled so that each hyperedge contains at most 2 colors, and instances where some hyperedge must contain all $\mathrm{k}$ colors. We prove that it is in fact NP-hard to decide whether there is a feasible labeling such that every hyperedge contains at most 2 colors, for $\mathrm{k}=3$.

\section{PRELIMINARIES}

A note on vector notation: We denote vectors in boldface, such as $\mathrm{v} \in \square^{\mathrm{k}}$. The coordinates of $\mathrm{v}$ are written in italics, such as $\mathrm{v}=\left(\mathrm{v}_{1}, \ldots, \mathrm{v}_{\mathrm{k}}\right)$. By $\mathrm{e}_{\mathrm{i}}$, we denote the canonical basis vectors $(0, \ldots, 1, \ldots 0)$.

\subsection{The Simplex-Lattice Hypergraph and sub-divisions of the simplex.}

Let $\mathrm{q} \geq 1$ be an integer and consider the $(\mathrm{k}-1)$ dimensional simplex defined by

$\Delta_{\mathrm{k}, \mathrm{q}}=\left\{\mathrm{x}=\left\{\mathrm{x}_{1}, \mathrm{x}_{2}, \ldots, \mathrm{x}_{\mathrm{k}}\right\} \in \square^{\mathrm{k}}: \mathrm{x} \geq 0, \sum_{\mathrm{i}=1}^{\mathrm{k}} \mathrm{x}_{\mathrm{i}}=\mathrm{q}\right\}$.

\subsection{The Simplex-Lattice Hypergraph.}

We consider a vertex set of all the points in $\Delta_{\mathrm{k}, \mathrm{q}}$ with integer coordinates:

$\mathrm{V}_{\mathrm{k}, \mathrm{q}}=\left\{\mathrm{a}=\left\{\mathrm{a}_{1}, \mathrm{a}_{2}, \ldots ., \mathrm{a}_{\mathrm{k}}\right\} \in \square^{\mathrm{k}}: \mathrm{a} \geq 0, \sum_{\mathrm{i}=1}^{\mathrm{k}} \mathrm{a}_{\mathrm{i}}=\mathrm{q}\right\}$.

3.3 The Simplex-Lattice Hypergraph is a k-uniform hypergraph

$\mathrm{H}_{\mathrm{k}, \mathrm{q}}=\left(\mathrm{V}_{\mathrm{k}, \mathrm{q}}, \mathrm{E}_{\mathrm{k}, \mathrm{q}}\right)$ whose hyperedges (which we also call cells due to their geometric interpretation) are indexed by $\mathrm{b} \in \square_{+}^{\mathrm{k}}$ such that $\sum_{\mathrm{i}=1}^{\mathrm{k}} \mathrm{b}_{\mathrm{i}}=\mathrm{q}-1$ : we have

$$
E_{k, q}=\left\{e(b): b \in \square^{k}, b \geq 0, \sum_{i=1}^{k} b_{i}=q-1\right\}
$$

where $e(b)=\left\{b+e_{1} ; b+e_{2} ; \ldots, b+e_{k}\right\}=\left\{\left(b_{1}+1, b_{2}, \ldots\right.\right.$ ., $\left.\left.b_{k}\right),\left(b_{1}, b_{2}+1, \ldots, b_{k}\right), \ldots,\left(b_{1}, b_{2}, \ldots, b_{k+1}\right)\right\}$. We sometimes omit the indices $\mathrm{k}$; $\mathrm{q}$ when there is no danger of confusion. For each vertex $a \in V_{k, q}$, we have a list of admissible colors $\mathrm{L}(\mathrm{a})$, which is

$$
\mathrm{L}(\mathrm{a})=\left\{\mathrm{i} \in[\mathrm{k}]: \mathrm{a}_{1}>0\right\}
$$

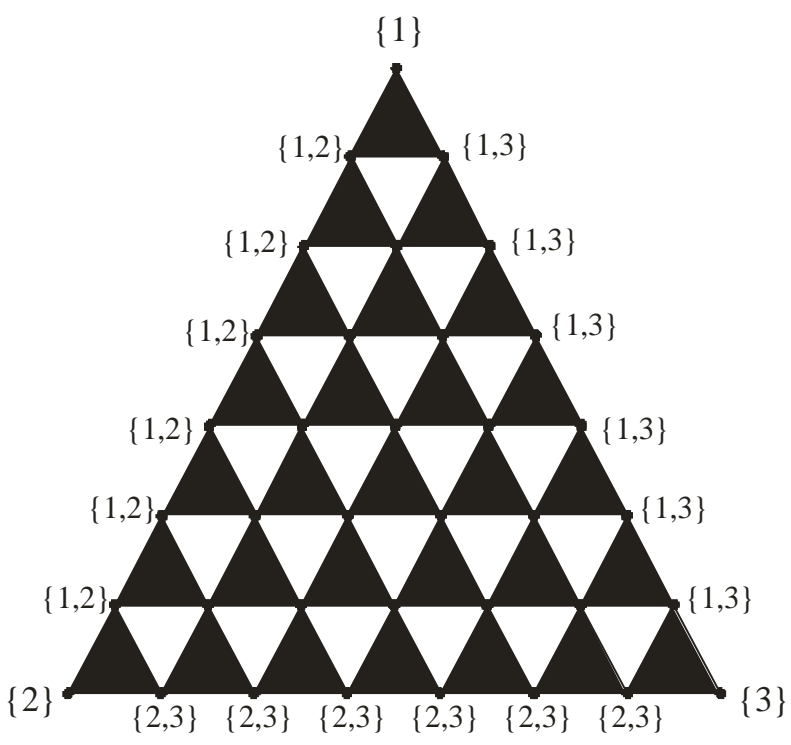

Figure 1: The Simplex Lattice Hypergraph for $\mathrm{k}=3 ; \mathrm{q}=$ 7 , with hyperedges shaded in gray. The gray triangles together with the white triangles form a simplicial subdivision. The lists of admissible colors are given on the boundary; for internal vertices the lists are all $\{1,2,3\}$.

The reader may notice that this is a setup reminiscent of Sperner's Lemma [7]. (See Figure 1.) However, Sperner's Lemma concerns subdivisions of the simplex; $\mathrm{H}_{\mathrm{k}, \mathrm{q}}$ is not a 
subdivision of the simplex since its hyperedges viewed as geometric cells do not cover the full volume of $\Delta_{\mathrm{k}, \mathrm{q}}$.

3.4 Simplicial subdivisions. A simplicial subdivision of $\Delta_{\mathrm{k}, \mathrm{q}}$ is a collection of simplices ("cells") $\Sigma$ such that

- The union of the cells in $\Sigma$ is the simplex $\Delta_{\mathrm{k}, \mathrm{q}}$.

- For any two cells $\sigma_{1}, \sigma_{2} \in \Sigma$, their intersection is either empty or a full face of a certain dimension shared by $\sigma_{1}, \sigma_{2}$.

We describe a concrete subdivision of $\Delta_{\mathrm{k}, \mathrm{q}}$ in Section 6 .

\section{COLORINGS OF SIMPLICIAL SUBDIVISIONS}

First, let us recall the statement of Sperner's Lemma [7]. We call a labeling $1: \mathrm{V} \rightarrow[\mathrm{k}]$ Sperner-admissible if $1(\mathrm{a}) \in$ $\mathrm{L}(\mathrm{a})$ for each $\mathrm{a} \in \mathrm{V}$; i.e., if $\mathrm{l}(\mathrm{a})=\mathrm{j}$ then $\mathrm{a}_{\mathrm{j}}>0$.

\subsection{Sperner's Lemma}

For every Sperner-admissible labeling of the vertices of a simplicial subdivision of $\Delta_{\mathrm{k}, \mathrm{q}}$, there is a cell whose vertices receive all $\mathrm{k}$ colors.

We remark that this does not say anything about the Simplex-Lattice Hypergraph: Even if the subdivision uses the point set $\mathrm{V}_{\mathrm{k}, \mathrm{q}}$, the rainbow cell given by Sperner's Lemma might not be a member of $\mathrm{E}_{\mathrm{k}, \mathrm{q}}$ since $\mathrm{E}_{\mathrm{k}, \mathrm{q}}$ consists only of scaled copies of $\Delta_{\mathrm{k}, \mathrm{q}}$ without rotation; it is not a full subdivision of the simplex. (See Figure 2.)

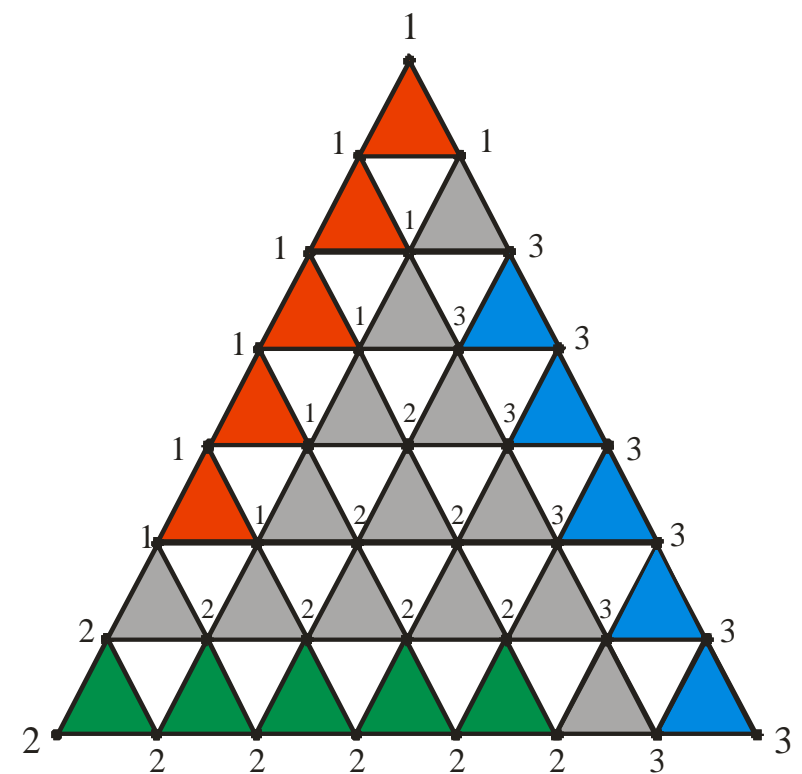

Figure 2: A Sperner-admissible labeling for $\mathrm{k}=3$ and $\mathrm{q}=$ 7. The set $\mathrm{E}$ of hyperedges consists of the shaded triangles. The gray triangles are non-monochromatic hyperedges. At least one triangle (not necessarily in E) must be 3-colored (rainbow). Instead of rainbow cells, the statement proposed (and proved for $\mathrm{k}=3$ ) in [3] involves nonmonochromatic cells.

\subsection{Statement: Proposition (Simplex-Lattice Coloring Lemma)}

For any Sperner-admissible labeling $1: \mathrm{V}_{\mathrm{k}, \mathrm{q}} \rightarrow[\mathrm{k}]$, there are at least $\left(\begin{array}{c}\mathrm{q}+\mathrm{k}-3 \\ \mathrm{k}-2\end{array}\right)$ hyperedges e $\in \mathrm{E}_{\mathrm{k}, \mathrm{q}}$ that are nonmonochromatic under 1 .

The first-choice labeling. In particular, the proposition is that a Sperner-admissible labeling minimizing the number of non-monochromatic cells is a first-choice one which labels each vertex a by the smallest coordinate $i$ such that $a_{i}>0$. Under this labeling, all the hyperedges $e(b)$ such that $b_{1}>0$ are labeled monochromatically by 1 . The only hyperedges

that receive more than 1 color are those where $b_{1}=0$, and the number of such hyperedges is exactly $\left(\begin{array}{c}q+k-3 \\ k-2\end{array}\right)$.

Proof. Consider the set of hyperedges $\mathrm{E}_{\mathrm{k}, \mathrm{q}}$ : observe that it can be written naturally as

$$
\mathrm{E}_{\mathrm{k}, \mathrm{q}}=\left\{\mathrm{e}(\mathrm{b}): \mathrm{b} \in \mathrm{V}_{\mathrm{k}, \mathrm{q}-1}\right\}
$$

i.e., the hyperedges can be identified one-to-one with the vertices in Vk,q-1. Recall that

$e(b)=\left\{b+e_{1} ; b+\right.$ $\left.e_{2}, \ldots, b+e_{k}\right\}$. Two hyperedges e $(b), e\left(b^{\prime}\right)$ share a vertex if and only if $\quad b^{\prime}+e_{j}=b+e_{i}$ for some pair $i, j \in[k]$; or in other words if $b, b^{\prime}$ are nearest neighbors in Vk,q-1 (differ by \pm 1 in exactly two coordinates).

Consider a labeling $1: \mathrm{V}_{\mathrm{k}, \mathrm{q}} \rightarrow[\mathrm{k}]$. For each $\mathrm{i} \in[\mathrm{k}]$, let $\mathrm{C}_{\mathrm{i}}$ denote the set of points in $\mathrm{V}_{\mathrm{k}, \mathrm{q}-1}$ representing the monochromatic hyperedges in color $\mathrm{i}$,

$\mathrm{C}_{\mathrm{i}}=\left\{\mathrm{b} \in \mathrm{V}_{\mathrm{k}, \mathrm{q}-1}: \forall \mathrm{v} \in \mathrm{e}(\mathrm{b}): \mathrm{l}(\mathrm{v})=\mathrm{i}\right\}$

Define an injective mapping $\phi_{\mathrm{i}}: \mathrm{C}_{\mathrm{i}} \rightarrow \mathrm{V}_{\mathrm{k}, \mathrm{q}-2}$ as follows: $\phi_{\mathrm{i}}(\mathrm{b})=\mathrm{b}-\mathrm{e}_{\mathrm{i}}$

The image is indeed in $\mathrm{V}_{\mathrm{k}, \mathrm{q}-2}$ : if $\mathrm{b} \in \mathrm{C}_{\mathrm{i}}$, we have $\mathrm{b}_{\mathrm{i}}>0$, or else $\mathrm{e}(\mathrm{b})$ would contain a vertex a such that $\mathrm{a}_{\mathrm{i}}=0$ and hence e(b) could not be monochromatic in color $\mathrm{i}$.

Therefore, $\mathrm{b}-\mathrm{e}_{\mathrm{i}} \in \square_{+}^{\mathrm{k}}$ and $\left(\mathrm{b}-\mathrm{e}_{\mathrm{i}}\right) \cdot 1=\mathrm{q}-2$ which means $\mathrm{b}-\mathrm{e}_{\mathrm{i}} \in \mathrm{V}_{\mathrm{k}, \mathrm{q}-2}$.

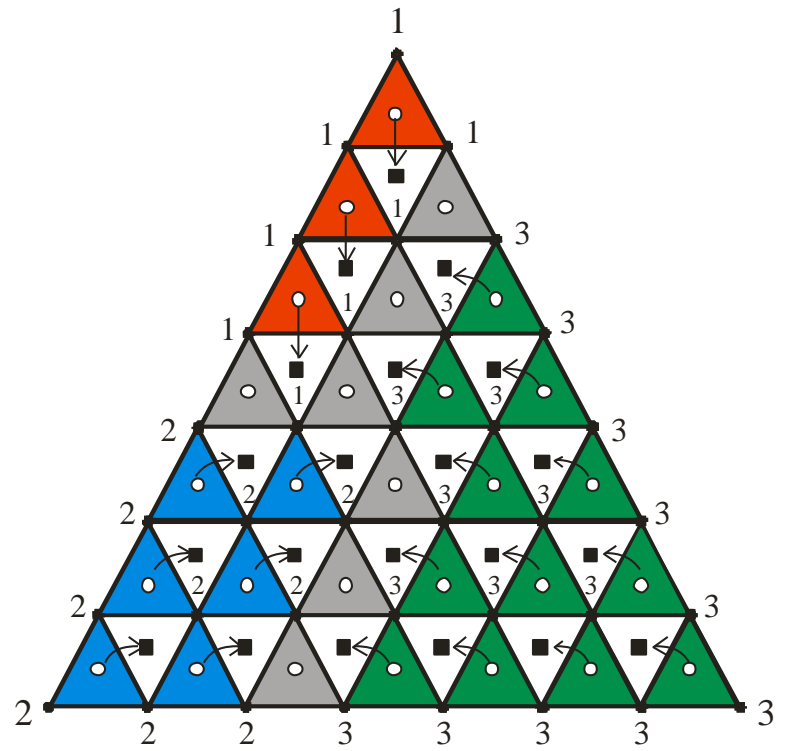

Figure 3 : The mappings $\phi_{\mathrm{i}}: \mathrm{C}_{\mathrm{i}} \rightarrow \mathrm{V}_{\mathrm{k}, \mathrm{q}-2}$. The hyperedges are represented by the empty circles; $\mathrm{C}_{\mathrm{i}}$ is the subset of 
them monochromatic in color i. The black squares coordinates" of a to be Top(a) $=(\pi(1), \ldots, \pi(t(a)))($ an represent $\mathrm{V}_{\mathrm{k}, \mathrm{q}-2}$; note that each point in $\mathrm{V}_{\mathrm{k}, \mathrm{q}-2}$ is the image ordered set).

of at most one monochromatic hyperedge.

Further, we claim that $\phi_{\mathrm{i}}\left[\mathrm{C}_{\mathrm{i}}\right] \cap \phi_{\mathrm{j}}\left[\mathrm{C}_{\mathrm{j}}\right]=\phi$ for every $\mathrm{i} \neq \mathrm{j}$. If not, there would be $b \in C_{i}$ and $b^{\prime} \in C_{j}$ such that $b-e_{i}=b^{\prime}$ - $e_{j}$. Then, the point $a=b+e_{j}=b^{\prime}+e_{i}$ would be an element of

both the hyperedge $\mathrm{e}(\mathrm{b})$ and the hyperedge $\mathrm{e}\left(\mathrm{b}^{\prime}\right)$. This contradicts the assumption that $\mathrm{e}(\mathrm{b})$ is monochromatic in color $\mathrm{i}$ and $\mathrm{e}\left(\mathrm{b}^{\prime}\right)$ is monochromatic in color $\mathrm{j}$. So the sets $\phi_{\mathrm{i}}\left[\mathrm{C}_{\mathrm{i}}\right]$ are pairwise disjoint subsets of $\mathrm{V}_{\mathrm{k}, \mathrm{q}-2}$. By the definition of $\phi_{\mathrm{i}}$, we clearly have $\left|\phi_{\mathrm{i}}\left[\mathrm{C}_{\mathrm{i}}\right]\right|=\left|\mathrm{C}_{\mathrm{i}}\right|$. We conclude that the total number of monochromatic hyperedges is

$$
\sum_{\mathrm{i}=1}^{\mathrm{k}}\left|\mathrm{C}_{\mathrm{i}}\right|=\sum_{\mathrm{i}=1}^{\mathrm{k}}\left|\phi_{\mathrm{i}}\left[\mathrm{C}_{\mathrm{i}}\right]\right| \leq\left|\mathrm{V}_{\mathrm{k}, \mathrm{q}-2}\right|
$$

The total number of hyperedges is $\left|E_{k, q}\right|=\left|V_{k, q-1}\right|$ Considering that $\left|\mathrm{V}_{\mathrm{k}, \mathrm{q}}\right|=\left(\begin{array}{c}\mathrm{q}+\mathrm{k}-3 \\ \mathrm{k}-2\end{array}\right)$ (the number of partitions of $\mathrm{q}$ into a sum of $\mathrm{k}$ nonnegative integers), we obtain that the number of non-monochromatic hyperedges is

$$
\left|E_{k, q}\right|-\sum_{i=1}^{k}\left|C_{i}\right| \geq\left|V_{k, q-1}\right|-\left|V_{k, q-2}\right|=\left(\begin{array}{c}
q+k-2 \\
k-1
\end{array}\right)-\left(\begin{array}{c}
q+k-3 \\
k-1
\end{array}\right)=\left(\begin{array}{c}
q+k-3 \\
k-2
\end{array}\right)
$$

\section{A LABELING OF $\mathrm{H}_{\mathrm{k}, \mathrm{q}}$ WITH AT MOST 4 COLORS ON EACH HYPEREDGE}

We recall that Sperner's lemma states that any Sperneradmissible labeling of a subdivision of the simplex must contain a simplex with all $\mathrm{k}$ colors. The hypergraph $\mathrm{H}_{\mathrm{k}, \mathrm{q}}$ defined in Section 3.1 is not a subdivision since it covers only a subset of the large simplex. It is easy to see that the conclusion of Sperner's lemma does not hold for $\mathrm{H}_{\mathrm{k}, \mathrm{q}}$ - for example for $\mathrm{k}=3$, we can label a 2-dimensional triangulation so that exactly one triangle has 3 different colors, and this triangle is not in $\mathrm{E}_{3, \mathrm{q}}$. (See Figure 2.) Hence, each triangle in $\mathrm{E}_{3, \mathrm{q}}$ has at most 2 colors. By an extension of this argument, we can label $\mathrm{H}_{\mathrm{k}, \mathrm{q}}$ so that each hyperedge in $E_{k, q}$ contains at most $\mathrm{k}-1$ colors. The question we ask in this section is, what is the minimum $1^{*}$ such that there is a Sperner-admissible labeling with at most $1^{*}$ colors on each hyperedge in $\mathrm{E}_{\mathrm{k}, \mathrm{q}}$ ? We prove the following result.

Lemma 5.1. For any $k \geq 4$ and $q \geq k^{2}$, there is a Sperneradmissible labeling of $\quad \mathrm{H}_{\mathrm{k}, \mathrm{q}}=\left(\mathrm{V}_{\mathrm{k}, \mathrm{q}}, \mathrm{E}_{\mathrm{k}, \mathrm{q}}\right)$ such that every hyperedge in $\mathrm{E}_{\mathrm{k}, \mathrm{q}}$ contains at most 4 colors.

Proof. We define a labeling $1: \mathrm{V}_{\mathrm{k}, \mathrm{q}} \rightarrow[\mathrm{k}]$ as follows:

- $\quad$ Given $a \in \mathrm{V}_{\mathrm{k}, \mathrm{q}}$, let $\pi:[\mathrm{k}] \rightarrow[\mathrm{k}]$ be a permutation such that $a_{\pi(1)} \geq a_{\pi(2)} \geq \ldots a_{\pi(k)}$ (and if $a_{\pi(i)}=a_{\pi(i+1)}$, we order $\pi$ so that $\pi(i)<\pi(i+1))$.

- $\quad$ Define $\mathrm{t}(\mathrm{a})$ to be the maximum $\mathrm{t} \in[\mathrm{k}]$ such that $\forall 1 \leq \mathrm{j} \leq \mathrm{t}, \mathrm{a}_{\pi(\mathrm{j})} \geq \mathrm{k}-\mathrm{j}+1$. We define the "Top
- We define the color of a to be $1(\mathrm{a})=\pi(\mathrm{t}(\mathrm{a}))$, the index of the "last Top coordinate".

First, we verify that this is a well-defined Sperner admissible labeling.

Since $\sum_{\mathrm{i}=1}^{\mathrm{k}} \mathrm{a}_{\mathrm{i}}=\mathrm{q} \geq \mathrm{k}^{2}$, we have $\mathrm{a}_{\pi(1)}=\max \mathrm{a}_{\mathrm{i}} \geq \mathrm{k}$ and hence $1 \leq \mathrm{t}(\mathrm{a}) \leq \mathrm{k}$. For each $\mathrm{a} \in \mathrm{V}_{\mathrm{k}, \mathrm{q}}$, we have $: \mathrm{a}_{\mathrm{l}(\mathrm{a})}=\mathrm{a}_{\pi(\mathrm{t}(\mathrm{a}))}$ $\geq \mathrm{k}-\mathrm{t}(\mathrm{a})+1>0$, since $\mathrm{t}(\mathrm{a}) \leq \mathrm{k}$. Therefore, 1 is Sperneradmissible.

\section{CONCLUSIONS}

We have proved several results about colorings of a discretization of the simplex. Our result can be viewed as being at the opposite end of the spectrum from Sperner's Lemma: Instead of the existence of a rainbow cell, we prove a lower bound on the number of nonmonochromatic cells. Due to the motivating Hypergraph Labeling problem, we consider a special hypergraph embedded in the simplex rather than a full subdivision. A natural question is whether an analogous statement holds for simplicial subdivisions. More generally, we might "interpolate" between Sperner's Lemma and our result and some assumption of regularity would be needed to obtain a general result

\section{REFERENCES}

[1] Chandra Chekuri and Alina Ene. Submodular cost allocation problem and applications. In Proc. of ICALP, 354\{366, 2011.

[2] Chandra Chekuri and Alina Ene. Approximation algorithms for submodular multiway partition. In Proc. of IEEE FOCS, 807-816, 2011.

[3] Alina Ene and Jan Vondrak. Hardness of submodular cost allocation: Lattice matching and a simplex coloring conjecture. In Proc. of APPROX, 144\{159, 2014.

[4] Alina Ene, Jan Vondrak, and Yi Wu. Local distribution and the symmetry gap: Approximability of multiway partitioning problems. In Proc. of ACM-SIAM SODA, 306-325, 2013.

[5] Jon Kleinberg and Eva Tardos. Approximation algorithms for classification problems with pairwise relationships: Metric labeling and Markov random elds. Journal of the ACM, 49(5):616\{639, 2002

[6] Rajsekar Manokaran, Joseph Naor, Prasad Raghavendra, and Roy Schwartz. SDP gaps and UGC hardness for multiway cut, 0extension, and metric labeling. In Proc. of ACM STOC, 11-20, 2008.

[7] Emanuel Sperner. Neuer Beweis f $\square$ ur die Invarianz der Dimensionszahl und des Gebietes. Math. Sem. Univ. Hamburg, 6:265-272, 1928

[8] Francis Edward Su. Rental harmony: Sperner's lemma in fair division. Amer. Math. Monthly, 106:930-942, 1999. 